\title{
Properties of the recombination of one-dimensional motion signals into a pattern motion signal
}

\author{
FRANK L. KOOI, KAREN K. DE VALOIS, DAVID H. GROSOF, and RUSSELL L. DE VALOIS \\ University of California, Berkeley, California
}

\begin{abstract}
We have examined the human ability to determine the direction of movement of a variety of plaid patterns. The plaids were composed of two orthogonal sine-wave gratings. When the plaid components are of unequal spatial frequency or sometimes of unequal contrast, observers judge the direction of movement incorrectly. In terms of the two-stage model of Adelson and Movshon (1982), these errors may result from either a misjudgment in the perceived speeds of each of the components or a failure in the combination of one-dimensional component movements into a coherent direction of motion of the two-dimensional plaid pattern, or both. A comparison of the perceived direction of motion of plaids with the relative perceived speeds of the plaid component gratings suggests that both failures occur, but in different circumstances. The relative perceived speed of the plaid components was measured with a spatial and a temporal forced-choice technique, the former leading to larger differences. Our results support the notion that the visual system decomposes a moving plaid into oriented components and subsequently recombines the component motions.
\end{abstract}

A considerable amount of psychophysical, physiological, and anatomical evidence has accumulated to indicate that the initial stages of the visual system perform a local two-dimensional (2-D) spatial frequency filtering of visual stimuli (see R. L. De Valois \& K. K. De Valois, 1988, for a review). The physiological evidence, for instance, indicates that cells in striate cortex have receptive fields localized to a particular patch of visual space, and that they respond to a pattern within that local region only insofar as it has power in the particular orientation and spatial frequency range to which that cell is tuned. K. K. De Valois, R. L. De Valois, and Yund (1979) and later Movshon, Adelson, Gizzi, and Newsome (1986) showed that cells in striate cortex do not respond to the overall pattern direction, but rather that they respond to the spatial frequency components within the cells' spatiotemporal passbands. For example, in the case of patterns defined as the sum of two gratings, which they termed plaids, responses were predictable from the orientation of the two Fourier components, but not from the perceived direction of the pattern as a whole.

Such local 2-D spatial filtering of images is advantageous for many aspects of vision, but it also poses

The research reported in this paper was supported by Grant EY00014 from the National Eye Institute and Grant BNS 8819867 from the National Science Foundation. F. L. Kooi and D. H. Grosof were associated with the Physiological Optics and Neurobiology Groups, respectively, at the University of California, Berkeley. D. H. Grosof is presently at the Center for Neural Science, New York University. Correspondence should be addressed to F. L. Kooi, who is now at TNO Institute for Perception, Kampweg 5, 3769 DE Soesterberg, The Netherlands (e-mail: kooi@izf.tno.nl). problems-in particular, the problem of how the visual system detects the real direction of motion of complex patterns. Consider the case of the plaid composed of two sine gratings as shown in Figure 1. As the plaid is drifted to the right behind the aperture, the results of $K$. K. De Valois et al. (1979) and Movshon et al. (1986) indicate that V1 cells will instead respond to the diagonal component motions. The true direction of motion of the overall pattern is therefore not explicitly coded at this stage of visual processing.

It has been proposed (Adelson \& Movshon, 1982) that the filtering stage is followed by a second stage in which information from the filters is recombined to yield the true direction of motion. Following Fennema and Thompson (1979), Adelson and Movshon show that the velocity of any 2-D stimulus, such as the plaid, is fully determined and is given by the intersection of the two constraint lines belonging to the components (see Figure 1). The question remains whether or not the visual system actually makes use of such a process.

A possible physiological basis for the second stage has been reported by Movshon et al. (1986) and Rodman and Albright (1989) in area MT. Although many of the MT cells were similar to $\mathrm{V} 1$ cells in their responses to moving plaids, $30 \%$ were different in that they responded to the true direction of movement rather than to the individual components. Movshon et al. (1986) suggest that these pattern-movement-selective MT cells combine the oriented component motions to compute the overall direction of movement. Alternatively, MT cells may receive input from nonoriented V1 cells or from the pulvinar (Rodman, Gross, \& Albright, 1989), in which case a component-to-pattern transformation may be unnecessary. 


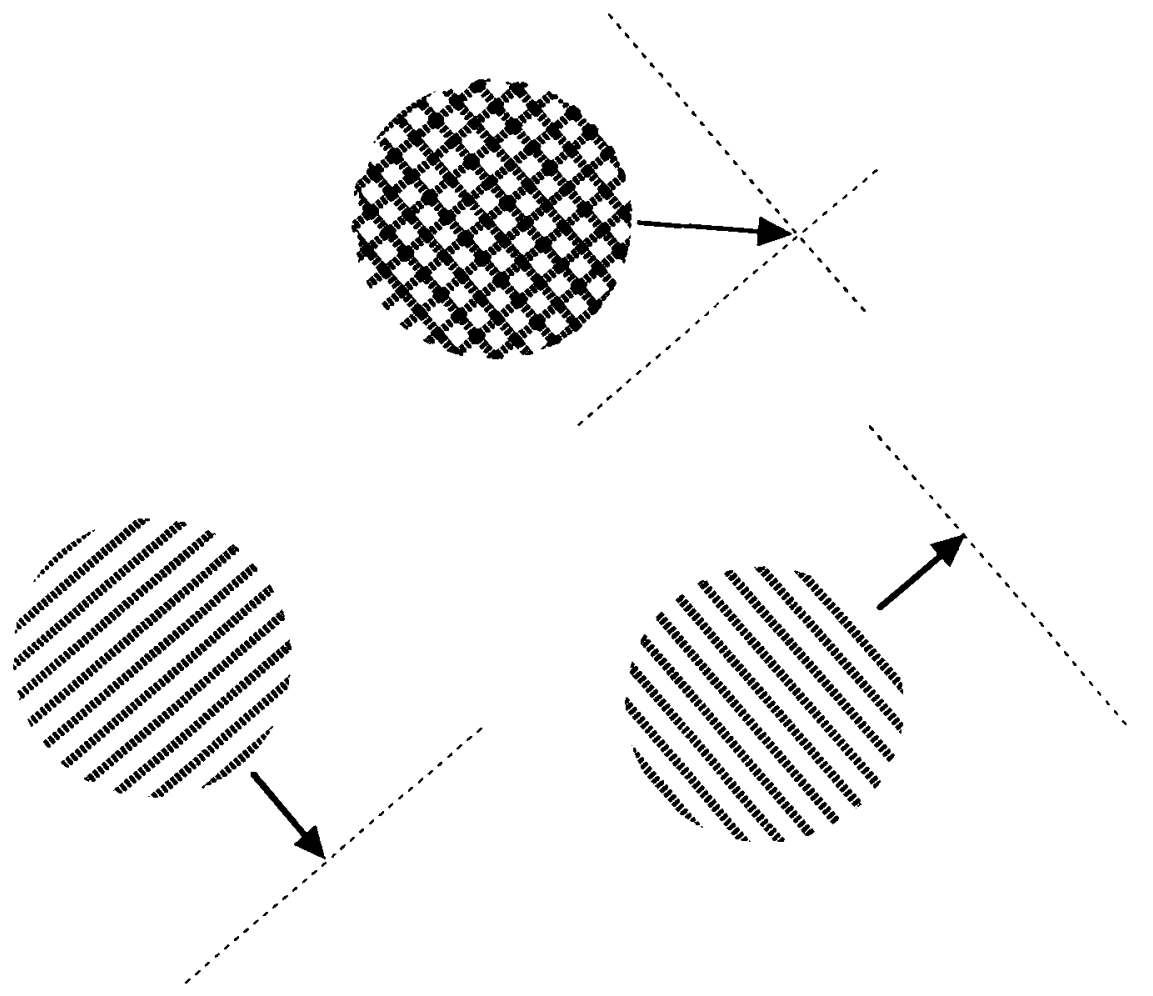

Figure 1. Schematic of the patterns used, the aperture problem, and the intersection-ofconstraints model for component recombination (adapted from Adelson \& Movshon, 1982, Figure 1). The top figure represents a plaid, the superposition of two gratings, separately represented below. The solid vectors represent the perceived velocities as each pattern is moved to the right. The dashed lines are constraint lines, representing the family of motions that are indistinguishable since only motion perpendicular to the grating orientation results in a change in position. For the plaid (top), the point of intersection is the single velocity that is consistent with both sets of constraints.

Psychophysical support for the existence of a second stage has come from various sources. The "sliding" percept of a moving plaid, in which the two components appear to move independently (Adelson \& Movshon, 1982; Kooi, De Valois, Switkes, \& Grosof, in press; Welch \& Bowne, 1990), is likely to reflect the output from the first stage under conditions that do not allow the recombination to occur. Component and pattern motion exhibit differential adaptation (Movshon et al., 1986) and masking (Ferrera \& Wilson, 1987). Welch (1989) showed that the speed discrimination threshold for a coherently moving plaid is limited by the orientation component stage, suggesting that the two stages are serial rather than parallel.

Several studies have shown that the perceived direction of a moving plaid may deviate from the actual direction of motion. The perceived direction is dominated by the component of higher contrast (Stone, Watson, \& Mulligan, 1990) and lower spatial frequency (Smith \& Edgar, 1991). Derrington and Suero (1991) examined perceived plaid direction as a function of motion adaptation. The existence of such perceived direction errors places constraints on models of 1-D component to 2-D pattern motion perception. Each study suggests that the error in perceived plaid direction is linked to the perceived speeds of the com- ponents. The latter two studies quantitatively tested the hypothesis by measuring the perceived component speeds and by applying the intersection of constraints model (Adelson \& Movshon, 1982) to them. In this way, the errors in perceived plaid direction could be accounted for.

We have further tested the recombination of 1-D motion signals into a pattern motion signal by examining the perceived direction of moving plaids, with components differing in both contrast and spatial frequency, at three eccentricities. The motion system has been shown to be disproportionately sensitive to lower spatial frequencies (Ramachandran \& Cavanagh, 1987; Smith \& Edgar, 1990), especially in the peripheral visual field (Ramachandran, 1987). This leads one to expect the same also to be the case for the perceived direction of moving plaids. We directly compare some of these perceived plaid direction errors with the perceived speeds of the individual components, as a test of the intersection of constraints model. The perceived speeds were measured with two different techniques (spatial and temporal forced choice), yielding different results and clarifying some of the confusion in the literature about perceived speed. A unique measure of "perceived speed" therefore does not seem to exist. Stone and Thompson (1992) recently reached the same conclu- 
sion. In another paper (Kooi \& K. K. De Valois, 1992), we tested the same conditions for equiluminant and colored patterns such that the results for luminance and color could be directly compared. These results have been presented in short form elsewhere (Kooi, K. K. De Valois, Grosof, \& R. L. De Valois, 1988; Kooi, R. L. De Valois, \& Wyman, 1988).

\section{GENERAL METHODS}

\section{Apparatus}

Stimuli were generated by a Data General Nova $4 X$ computer with a Lexidata graphics unit, as described more fully elsewhere (Switkes, Bradley, \& K. K. De Valois, 1988), and displayed on a Mitsubishi RGB monitor. The plaid patterns were made up of two 1-cycle/degree (cpd) sine-wave gratings oriented at $45^{\circ}$ and $135^{\circ}$ relative to horizontal, forming a sine-sine plaid. The stimuli were $2.5^{\circ}$ in size. For the foveal presentations, a small fixation dot was placed in the center of the viewing circle in the middle of a small translucent diffuser patch made of tracing paper (radius $=0.67^{\circ}$ ) that transmitted most of the light but little of the underlying pattern structure. The stimulus region was surrounded by a diffuser ring with a square drawn upon it to serve as a reference for vertical and horizontal. The space-averaged luminance of the display was $27.4 \mathrm{~cd} / \mathrm{m}^{2}$, with diffuse background lighting in the room. The mean chromaticity was yellow (CIE $x=0.504$, and $y=0.434$ ).

\section{Spatiotemporal and Contrast Limitations}

The $60-\mathrm{Hz}$ interlaced monitor had a spatial resolution of 26 pixels/ $\mathrm{deg}$ at the $172-\mathrm{cm}$ viewing distance. Continuous motion was approximated by small jumps. For low-contrast luminance plaids, the small resultant positional jumps were invisible and the motion appeared smooth. In addition to the intensity resolution of 8 bits of the graphics unit, which allowed the simultaneous presentation of 256 intensity levels, the average contrast could be adjusted by means of an added attenuator that operated under computer control. Thus, a full range of contrasts, including those near threshold, could be accurately displayed.

\section{Subjects}

Six experienced subjects, three of whom (S.L., M.B., and S.S.) were naive about the purpose of the particular experiments, participated in the experiment. Three subjects had normal vision; the other 3 subjects (F.K., D.G., and S.S.) had corrected-to-normal vision.

\section{Sensitivity Measurements}

To scale the various plaid components in terms of visibility or multiples of threshold contrast, we measured each subject's contrast sensitivity for each of the sine-wave grating components used in the plaids. The procedure was the same as that described by Switkes et al. (1988). Using a temporal two-alternative, forcedchoice (2AFC) staircase method converging on 75\% correct, thresholds were measured with a temporally Gaussian-ramped presentation of gratings drifting at $3 \mathrm{~Hz}$. The rise and fall times were $100 \mathrm{msec}$, and the plateau was $500 \mathrm{msec}$. Feedback was provided. These measurements allowed us to scale the results of subsequent experiments in terms of multiples of threshold contrasts. ${ }^{\mathrm{I}}$

\section{EXPERIMENT 1}

\section{Effect of Contrast, Spatial Frequency, and Retinal Location on the Perceived Direction of Plaids}

\section{Method}

A drifting plaid pattern was presented, and the subject was asked to report the apparent direction of motion. Stimuli were randomized with respect to both the direction of motion and the type of plaid presented. The predominant, baseline direction of motion was randomly chosen to be up, down, to the left, or to the right in order to avoid directional adaptation or an expectation of a certain direction of motion.

On any particular trial, the actual direction of motion of the plaid differed from one of these baseline directions by a small angle. Within a given session, six different small angles of direction of motion were presented in random order (method of constant stimuli). Each of these could be added to any of the four basic directions. Thus, there could be any of six small deviations from horizontal movement to the right, for instance, or six small deviations from vertical upward movement. The speed of the plaid was kept constant. On each trial, the subject reported whether the plaid moved toward the first or third quadrant (1-3 quad) or, alternatively, toward the second or fourth quadrant (2-4 quad). The subjects did not receive feedback.

To reduce an observer's ability to recognize the patterns, we employed two mirror images of the same plaid in each block. From trial to trial, this stimulus variable of plaid version was randomly varied in addition to the small angle and the basic direction. In total, each trial session contained six directions, two plaid versions (the mirror images), and five trials per condition, yielding a total of 60 trials per set. ${ }^{2}$

To reduce contamination of the motion of the pattern by an onset transient the plaid was presented in a stationary position for $333 \mathrm{msec}$ before the pattern began to move. In preliminary experiments, we found no significant effect of movement duration on our measures of movement direction over a range of 333 to $1,300 \mathrm{msec}$. The plaids were drifted for $667 \mathrm{msec}$ at $4.24 \% \mathrm{sec}$.

The subject's responses for the two mirror-image conditions and the four main directions (up, down, left, right) were averaged. Patterns of results for two mirror versions should be opposites of each other. Any directional bias not dependent on the difference between the sine-wave components, such as a subject's tendency to prefer one pair of quadrants over another, was thereby averaged out.

Responses for the direction angles were tabulated in a psychometric function (Figure 2). Perceived-direction responses are plotted versus the actual direction (angle) of plaid motion. The dashed line indicates veridical responses. For the example given, the plaid appeared to move slightly off-axis in the direction of the higher contrast component, while it was actually moving on-axis. To make

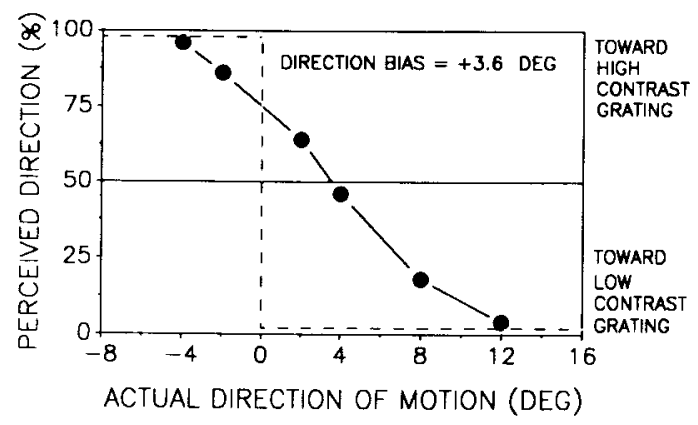

Figure 2. A representative psychometric function demonstrating the determination of the direction bias. The percentage of trials in which the plaid is seen moving in the direction of the higher contrast grating component is plotted vertically versus the actual direction of plaid motion. The dashed line indicates veridical responses. The direction bias is defined as the actual direction of motion for which the plaid is perceived to be moving exactly horizontally or vertically (on-axis). The exact location is determined by probit analysis. In the example given, the direction bias is computed to be $+3.6^{\circ}$. Typical standard errors are on the order of $0.4^{\circ}$. 
it appear on-axis, the physical pattern direction had to be shifted $+3.6^{\circ}$. This point was taken as the measure of the amount of perceptual "direction bias." The direction bias is defined as the angle necessary to make the apparent direction of the plaid on-axis. At still larger deviations from on-axis, the plaid appeared to move in the direction of the lower contrast component. The location of the crossover and the standard error were estimated by probit analysis (Finney, 1971) on at least five trial sets (a minimum of 300 measurements per data point) for the contrast and retinal location experiments and on at least three trial sets for the spatial frequency experiment. Typical standard errors, a measure of the confidence in the crossover estimate, were on the order of $0.4^{\circ}$.

\section{Results}

Contrast. The apparent direction of motion was measured for plaids with components identical in spatial frequency but different in contrast. The contrast ratio $\left(C_{1} / C_{2}\right)$ of the two components was fixed $(0.8)$, whereas the mean $\left[\left(C_{1}+C_{2}\right) / 2\right]$ of the contrasts was varied. Both components always were above threshold. In Figure 3, bias in perceived direction is plotted as a function of mean contrast. It can be seen that as the mean contrast increased, the size of the direction bias decreased. Thus, at the fixed contrast ratio (0.8), a lower mean contrast resulted in a larger perceived direction error. The direction bias became very small at a mean contrast of about $5 \%$ (with the exception that Subject M.B. showed a small residual effect), which is approximately 12 times threshold at these spatial and temporal frequencies. The perceived-direction error is therefore present only over a relatively small contrast range, demonstrating the robustness of the motion system with respect to contrast, in agreement with Stone et al. (1990). Similar contrast saturations occur in other types of motion experiments (motion aftereffect, motion hyperacuity, speed judgment accuracy, perceived speed, and direction discrimination: Campbell \& Maffei, 1981; Derrington \& Goddard, 1989; Keck, Palella, \& Pantle, 1976; McKee, Silverman, \& Nakayama, 1986; Nakayama \& Silverman, 1985).

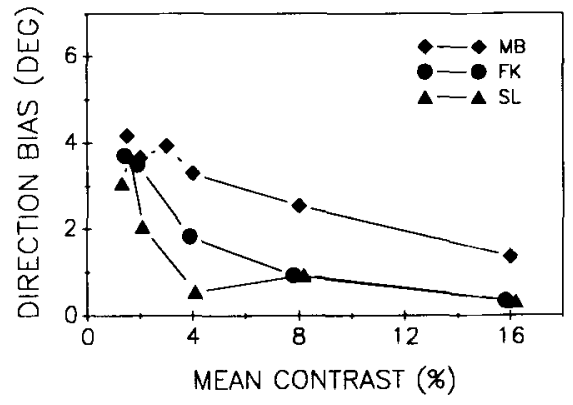

Figure 3. Direction bias for a luminance plaid with components of equal spatial frequencies ( $1 \mathrm{cpd})$ and unequal contrasts $\left(C_{1} / C_{2}\right.$ $=0.8$ ). Direction bias (in degrees) is plotted versus the average contrast of the two plaid components $\left[\left(C_{1}+C_{2}\right) / 2\right]$. The direction bias is our measure of the error in perceived direction of plaid motion. Positive direction bias indicates that the higher contrast component dominated the perceived direction of motion. Data are plotted for 3 subjects. Except for Subject M.B., significant direction bias occurs only below an average contrast of $5 \%$.

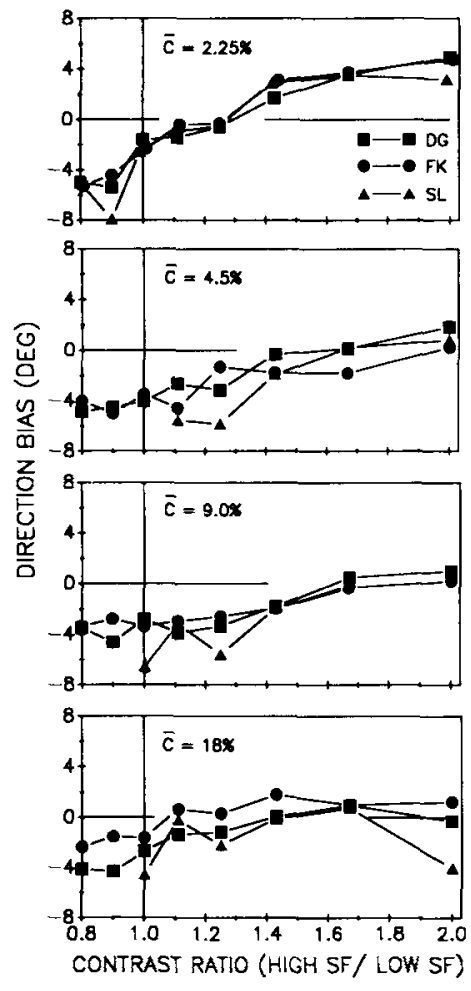

Figure 4. Direction bias for luminance plaids with unequal spatial frequencies. The components are 1 and $1.5 \mathrm{cpd}$. For the four graphs, the mean plaid contrasts $\left[C=\left(C_{1}+C_{2}\right) / 2\right]$ are $2.25 \%, 4.5 \%$, $9.0 \%$, and $18 \%$. For each graph, direction bias is plotted versus the contrast ratio of the higher spatial frequency to the lower spatial frequency component $\left(C_{1,3} / C_{1.0}\right)$. The three symbols indicate the 3 subjects. Positive direction bias indicates that the higher spatial frequency component dominated the perceived direction of motion. For equal multiples of threshold (contrast ratio near 0.95 ), all subjects show low-spatial-frequency dominance at each average contrast level. Only at the lowest average contrast (2.25\%) does the high spatial frequency ever significantly dominate.

Spatial frequency. The original experiment was repeated with plaid components of 1.0 and $1.5 \mathrm{cpd}$. The mean contrast was fixed at one of four levels, and the contrast ratio of the components was varied. (In the previous experiment, the contrast ratio was fixed and the mean contrast was varied.) At zero direction bias, both component speeds were $3 \% \mathrm{sec}$. The contrast sensitivities for these two gratings differ very little (Figure 7 shows data for 2 subjects). Averaged over all subjects, equal visibility (i.e., equal multiples of threshold) occurred near a contrast ratio of 0.95 .

The data from this experiment are plotted in Figure 4. The four graphs show the same experiment carried out at four different mean contrast levels. Direction bias is plotted versus the ratio of the high spatial frequency to the low-frequency component contrast. A positive direction bias indicates high-spatial-frequency dominance; a negative direction bias indicates low-spatial-frequency dominance. It can be seen that at equal multiples of thresh- 
old, the lower spatial frequency component clearly dominates the direction of motion of the plaid at all mean contrasts. Increasing the contrast ratio reduces the amount of the low-spatial-frequency dominance, but only at the lowest mean contrast $(2.25 \%)$ does the high spatial frequency ever dominate. The shallowness of the slopes for the higher mean contrasts is consistent with the lowcontrast saturation demonstrated in the previous experiment, which showed that above $5 \%$ average contrast the influence of a contrast difference is minimal. The lowspatial-frequency dominance, on the other hand, is mostly independent of average contrast.

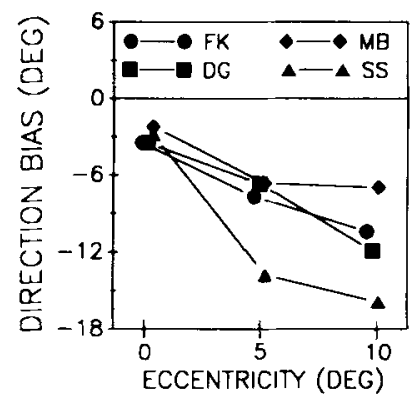

Figure 5. Direction bias as a function of eccentricity for unequal spatial frequency plaids (1- and 1.5-cpd components). For convenience, the condition in which the plaid is presented between $0.67^{\circ}$ and $2.5^{\circ}$ eccentricity is indicated by " 0 deg eccentricity." The average contrast is $4.5 \%$, and the contrast ratio 1.0. Data are plotted for 4 subjects. The amount of direction bias increases significantly with eccentricity for the unequal spatial frequency plaids.

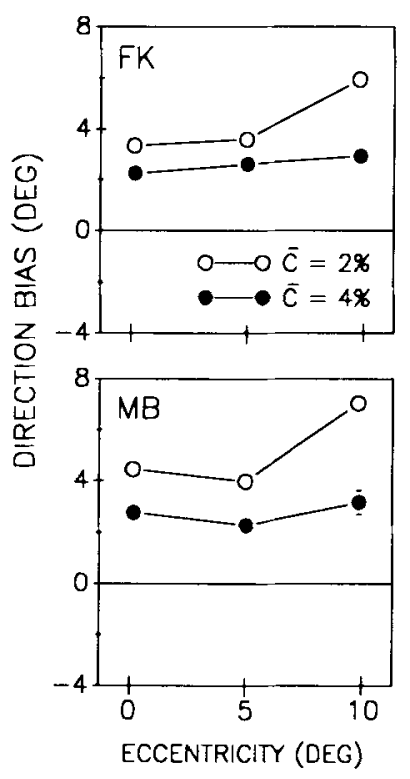

Figure 6. Direction bias as a function of eccentricity for unequal contrast plaids $\left(C_{1} / C_{2}=0.8\right)$. The mean contrasts are $2 \%$ and $4 \%$. Data are plotted separately for 2 subjects. Except for the $2 \%$ condition at $10^{\circ}$, only a minimal change in direction bias occurs as a function of eccentricity.

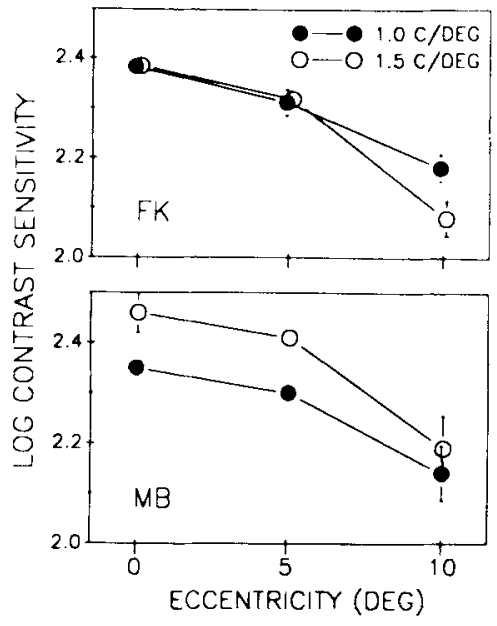

Figure 7. Contrast sensitivity of the plaid components. Log contrast sensitivity is plotted for 1 - and 1.5-cpd horizontal gratings as a function of eccentricity for 2 subjects.

Retinal location. Sample conditions from the previous experiments were repeated at two locations in the peripheral visual field. The stimulus was presented at $5^{\circ}$ and $10^{\circ}$ in the temporal visual field of the right eye. The small central fixation spot used for the previous experiments (here noted as the $0^{\circ}$, or foveal, condition) was left out. Using unequal spatial frequency plaids with $4.5 \%$ mean contrast and a contrast ratio of 1.0 , we found that the low-frequency component dominated perceived direction much more strongly at $5^{\circ}$ and $10^{\circ}$ (Figure 5) than it did foveally.

The direction bias due to a contrast difference, on the other hand, is not dependent on eccentricity (Figure 6). We tested unequal contrast plaids (contrast ratio $=0.8$ ) for the $2 \%$ and $4 \%$ mean contrast conditions. Only at the $2 \%$ mean contrast at $10^{\circ}$ did the direction bias rise significantly, probably due to the reduction in contrast sensitivity (see Figure 7). ${ }^{3}$

\section{EXPERIMENT 2}

\section{Relative Perceived Speed of the Plaid Components}

\section{Method}

Two psychophysical methods were used: spatial and temporal $2 \mathrm{AFC}$. The spatial or temporal position (left-right or first-second) was randomized from trial to trial. The spatial 2AFC technique has the two grating components presented simultaneously at the same orientation and drift direction, in neighboring apertures. The duration was $500 \mathrm{msec}$, with 100-msec on and off ramps. Each aperture was square, $4.1^{\circ}$ on each side, the two being separated by a $0.77^{\circ}$ strip. The subject was instructed to fixate between the two patches and was asked which of the two components appeared to move faster. The speeds varied around a mean of $3 \% \mathrm{sec}$. Threshold was determined by a 2 AFC staircase paradigm in which the relative speed of the two components was varied. At least eight staircases per condition were nun. Typical standard errors were on the order of $1.5 \%$.

The temporal $2 \mathrm{AFC}$ procedure involved presenting gratings in two successive 500 -msec intervals, separated by a blank period of $500 \mathrm{msec}$, in the same aperture configuration used for the directionbias experiments. The subject was again asked which of the two gratings appeared to move faster. A method of constant stimuli analo- 
gous to the direction-bias task was used. Instead of six different plaid directions, six different speed ratios were presented (the average speed was kept constant at $3 \% \mathrm{sec}$ ). The data analysis was the same as for the plaid-direction task.

\section{Results}

Contrast. For the unequal-contrast conditions, the higher contrast grating always appeared to be moving faster than the lower when matched for actual speed. In Figure 8, the perceived-speed ratio is plotted as a function of mean contrast. The largest mismatches were observed at the lower mean contrasts. The temporal technique (filled symbols) yielded noticeably smaller speed mismatches than did the spatial technique (open symbols). A simple change in technique thus can lead to a rather different estimate of perceived speed.

Spatial frequency and retinal location. The relative perceived speed of the 1- and 1.5-cpd gratings was measured for some of our subjects (Figure 9). With the spatial 2AFC technique (filled symbols), only small differences were found in the fovea. In the periphery, the lower spatial frequency grating gained noticeably in perceived speed, relative to the higher spatial frequency. With the temporal 2AFC technique (open symbols), perceived speed was measured only in the fovea. Data are shown for 4 subjects. For each, the higher spatial frequency appeared to move faster than the lower spatial frequency.

We do not have a firm explanation for why spatial and temporal 2AFC differ for both the contrast and spatialfrequency experiments, but we can offer some suggestions. Informally, it seemed easier to extract the true physical speed with the temporal 2AFC paradigm. Attention is necessarily divided with spatial 2 AFC but not with temporal 2AFC. Perhaps two mechanisms to determine speed exist: one attentional and the other not. With spatial 2AFC, the task had a different character; the most natural way

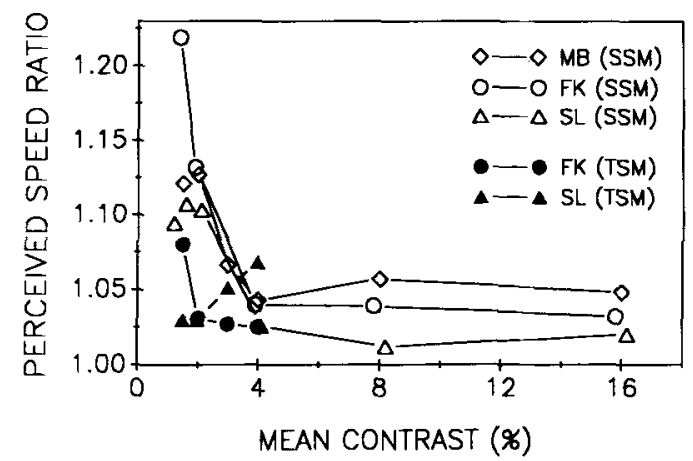

Figure 8. Speed-matching results for components differing in contrast $\left(C_{1} / C_{2}=0.8\right)$. The speed ratio that makes the two gratings appear to move equally fast is plotted as a function of the average contrast. A positive perceived-speed ratio indicates that the higher contrast grating appeared to move faster at equal speed. Open symbols = spatial speed matching (spatial 2AFC for 3 subjects). Closed symbols = temporal speed matching (temporal 2AFC for 2 subjects). Large perceived speed mismatches only occur at low mean contrast.

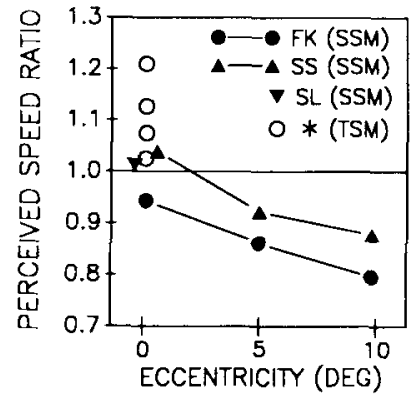

Figure 9. Speed-matching results for components differing in spatial frequency ( 1 and $1.5 \mathrm{cpd}$ ). The speed ratio that makes the two gratings appear to move equally fast is plotted as a function of eccentricity. A positive perceived-speed ratio indicates that the higher spatial frequency grating appeared to move faster at equal speed. Closed symbols = spatial speed matching ( 3 subjects). Open symbols $=$ temporal speed matching ( 4 subjects, only at $0^{\circ}$ eccentricity).

to match the two patterns was more according to the "momentum" than according to the speed of the gratings. The term "perceived motion strength" thus might be a more suitable choice than "perceived speed," even though the subjects were instructed to judge speed. In any case, the discrepancy in results suggests that the spatial and temporal techniques measure different properties.

The perceived velocity of a stimulus is dependent on the motion of the surrounding area (Duncker, 1938). This phenomenon of motion induction might account for some of the difference between the temporal and spatial paradigms. During a temporal presentation sequence, induction clearly cannot play a role since the grating stimulus is the only moving element of the display. However, with the spatial speed-matching technique, as well as with moving plaids, motion induction could play a role, because two moving elements are present in each case (side by side or orthogonally overlapping). The motion induction would have to be strongly dependent on contrast.

Previous experiments measuring perceived speed have yielded different and often conflicting outcomes. These studies may be reinterpreted in terms of the spatial versus temporal forced-choice distinction. On the one hand, highspatial-frequency slowdown has been reported (Smith \& Edgar, 1990); on the other hand, low-spatial-frequency slowdown has been found (Campbell \& Maffei, 1981; Diener, Wist, Dichgans, \& Brandt, 1976; Ferrera \& Wilson, 1991; McKee et al., 1986). The former study (Smith \& Edgar, 1990) used a spatial 2AFC paradigm; the latter studies temporally separated the stimuli in various ways. Campbell and Maffei (1981), using stimuli separated in both space and time, found low-contrast slowdown very similar to our spatial 2 AFC results. The contrast effect saturated near 5\%. Thompson (1982) also recorded lowcontrast slowdown with a spatial method of adjustment task. His results do not show as strong a low-contrast saturation, however. To conclude, perceived speed shows a strong but consistent dependence on the psychophysical measure used. 


\section{RELATION BETWEEN DIRECTION BIAS AND PERCEIVED COMPONENT SPEED}

\section{Theory}

The actual direction of motion of a $\pm 45^{\circ}$ plaid is linked to the relative speed of the two components:

$$
\tan (45-\theta)=S_{1} / S_{2} \text {. }
$$

where $S_{1}$ and $S_{2}$ are the speeds of the components and $\theta$ is the angular difference in degrees from the plaid direction when the speeds are equal. Similarly, a hypothetical relation between the perceived direction of the plaid (as measured by the direction bias) and the perceived speed of the components (indicated by lowercase $s_{1}$ and $s_{2}$ ) can be proposed:

$$
\tan (45-\text { direction bias })=s_{1} / s_{2} .
$$

The direction bias is the $\theta$ necessary to make the perceived plaid direction on-axis; the perceived speed ratio is the relative speed necessary to make the perceived speeds of the components equal. Our direction bias data can therefore also be viewed in terms of effective "motion strengths" of the components. Conversely, the measured perceived speeds of the components can be transformed to yield a predicted direction bias. For the following analysis, the relative perceived speeds measured in Experiment 2 have been transformed according to Equation 2 to yield predicted direction biases. The validity of the hypothesis can be tested by the degree of agreement between predicted and measured bias.

\section{Contrast}

In Figure 10, the measured biases from Figure 3 are replotted separately for each subject, together with the predicted biases based upon Equation 2 and our speed-matching results. The predicted biases are quite close to the spatial 2AFC speed-matching data for Subjects S.L. and F.K. and somewhat less so for Subject M.B., for whom smaller direction biases were predicted than were observed. Overall, the perceived-plaid-direction error correlates fairly well with the perceived-speed error as measured with the spatial $2 \mathrm{AFC}$ technique, suggesting that pattern direction may indeed be computed by Equation 2 (or a similar equation), with perceived component speed substituted for physical component speed. Biases may therefore arise from a faulty analysis of the speeds of the 1-D components rather than from a faulty recombination of the component signals.

\section{Spatial Frequency and Retinal Location}

In Figure 11, the measured biases from Figure 5 are replotted separately for 2 subjects, together with the predicted biases based upon Equation 2 and our spatial 2AFC speed-matching results from Figure 9. Predicted and measured direction biases do not match exactly, though both curves show increasing low-spatial-frequency dominance

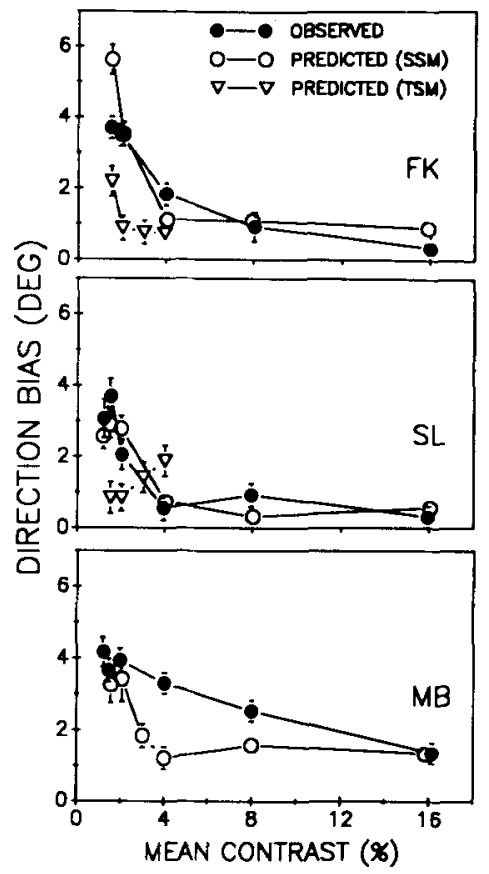

Figure 10. Comparison of perceived-speed and perceived-direction results for unequal contrast plaids. The direction bias data from Figure 3 are replotted separately for each subject, together with the predictions based upon the speed-matching results. The measured relative speed of the two components is converted into a predicted direction bias by Equation 2. Closed circles = perceived plaid direction as measured with the direction-bias technique. Open symbols $=$ expected or predicted direction biases based on the experimentally measured relative perceived speeds of the two plaid components. Open circles $=$ spatial 2AFC. Open triangles $=$ temporal 2AFC. With spatial 2AFC, the agreement is quite close for Subjects S.L. and F.K., and for Subject M.B. it is a little less so. Speed matching measured with temporal 2 AFC always significantly underestimated the expected direction biases.

with eccentricity. The perceived-speed measurements consistently underestimate the measured-direction bias. With foveal fixation, a direction bias exists even though the perceived speed of the two plaid components is nearly equal. The discrepancy is worse when plaid direction is compared with the temporal 2AFC data (Figure 9) and with most other speed-matching studies (Campbell \& Maffei, 1981; Diener et al., 1976; Ferrera \& Wilson, 1991; McKee et al., 1986), which show high-spatial-frequency speedup. On the other hand, Smith and Edgar (1991), who measured perceived plaid direction and perceived speed as a function of overall speed, reached the conclusion that the errors are well correlated. The lower spatial frequency was dominant in both cases, the deviations being largest at high temporal frequencies. For our conditions, however, the coarser component has an inherent advantage in determining plaid direction over determining perceived speed, suggesting that there may be more to determining plaid direction than the perceived speed of the components alone. 


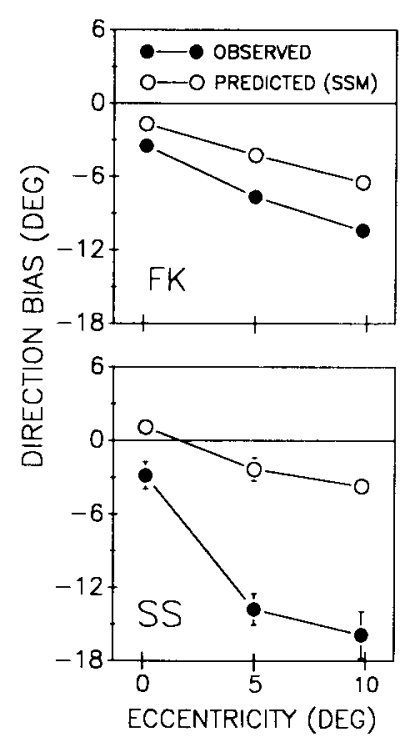

Figure 11. Comparison of perceived-speed and perceived-direction results for unequal spatial frequency plaids. The direction-bias data from Figure 5 are replotted separately for 2 subjects, together with the predictions based on the speed-matching results. The measured relative speed of the two components is converted into a predicted direction bias by Equation 2. Closed circles = perceived plaid direction as measured with the direction-bias technique. Open circles = expected direction biases based on the experimentally measured relative perceived speeds of the two plaid components. Both measured and predicted direction biases show more low-spatial-frequency dominance in the periphery. However, the two do not match quantitatively. The perceived direction of unequal spatial frequency plaids therefore cannot be completely accounted for by the perceived speeds of the components.

\section{DISCUSSION}

The percept of a sliding versus a coherent sine-wave plaid has been the subject of several psychophysical and theoretical studies. The main concerns have been to understand what determines whether the plaid appears coherent (Adelson \& Movshon, 1982; Kooi et al., in press; Krauskopf \& Farell, 1990; Welch \& Bowne, 1990), and what direction and speed is assigned to coherent plaids (Derrington \& Suero, 1991; Ferrera \& Wilson, 1990; Heeger, 1987; Kooi \& K. K. De Valois, 1992; Smith \& Edgar, 1991; Stone et al., 1990). The other goal is to elucidate the mechanism by which the components could be first decomposed and then recombined. We confirmed two factors that cause the perceived direction of motion to deviate from the real physical direction. A plaid's trajectory is biased toward the direction of the component of higher contrast or lower spatial frequency. The size of the latter advantage is dependent on the eccentricity.

\section{Relation Between Perceived Direction and Speed}

The reasonable agreement between measured- and predicted-direction biases due to a contrast difference (measured with spatial $2 \mathrm{AFC}$ ) indicates that the perceived direction of motion of a plaid can be accounted for by the per- ceived, rather than real, speeds of its components. The agreement indicates a common source for the two errors. The intersection-of-constraints model can be made to incorporate these results by letting it operate on perceived, rather than real, speed. This agreement supports the existence of a component-to-pattern transformation preceded by a Fourier-like decomposition and provides support for the intersection-of-constraints model as the recombination mechanism. Our results are also consistent with a vector summation model, because in our experiments the plaid components were always oriented perpendicularly. However, the inadequacy of vector summation is easy to demonstrate with plaids of other relative orientations (Movshon et al., 1986) and is not a realistic option for the component-to-pattern transformation.

A possible second source of plaid direction misperception, low-spatial-frequency dominance, appears to be independent of the direction bias due to contrast. In addition, for our limited conditions, this error can only partially be accounted for in terms of perceived component speed, suggesting that part of the "error" may be made in the component-to-pattern transformation itself: the lower spatial frequency appears to be weighted more heavily than one would expect on the basis of its speed. Given the results of Smith and Edgar (1991), who did find reasonable correlations between apparent plaid direction and perceived speed at other temporal frequencies, it is unclear whether this interpretation can be extrapolated to other spatial or temporal frequency combinations.

We suggest that the low-spatial-frequency dominance may be akin to the motion capture described by Ramachandran and Cavanagh (1987), in that the higher spatial frequency component appears to move along with the lower spatial frequency component. Our stimulus is different though in that the plaid is moving rigidly; the examples in the literature of motion capture all involve conflicting motion stimuli. One simple explanation for the motion capture we observe is inhibition or masking from low- onto high-spatial-frequency mechanisms at a stage preceding the component signal recombination stage. The motion signal from the low-spatial-frequency channel would thereby be weighted more heavily than that from the high-spatial-frequency channel, not only in perceiving plaids but also in experiments with compound 1-D gratings (Grosof, 1989).

The increase in low-spatial-frequency dominance with eccentricity, both in perceived plaid direction and relative perceived speed, is consistent with the increase of motion capture in the periphery reported by Ramachandran (1987). We suggest that the visual system assigns a stronger weight to low-spatial-frequency motion signals when the short-range motion system is adequately stimulated (e.g., in the present experiments), as well as when it is not (e.g., in Ramachandran \& Cavanagh, 1987).

An alternative explanation for the analysis of plaid direction is that the visual system detects the movement of the intersections ("blobs") rather than integrating the component motions. The direction biases could be due to the asymmetric profiles (shapes) of the nodes in the 
plaids (Perrone, 1990). Given the other evidence for the existence of a component stage and the reasonable explanation of the direction-bias data by the misperception of component speed, the interpretation offered above is more likely. The agreement between judged direction of motion and perceived speed shown in Figure 10 would have to be pure coincidence or would have to be the result of a mechanism relating component speed and pattern direction other than the simple one described in Equation 2. The strong correlation between perceived plaid direction and perceived component speed obtained with equiluminant plaids (Kooi \& K. K. De Valois, 1992) also strongly favors an initial decomposition and subsequent recombination of a plaid. However, the possibility that perceived plaid direction is determined from a combination of component and node motions is left open. Different experimental conditions might highlight one or the other.

\section{Sliding}

Adelson and Movshon (1982) showed that the amount of sliding in a plaid is dependent upon the contrast and spatial frequency difference between the two component gratings. The plaids with components of unequal contrast but equal spatial frequencies always appeared coherent. In order to maximize the coherence of the other plaids, the spatial frequency difference was chosen to be small, a factor of 1.5. Nevertheless, the unequal spatial frequency plaids did exhibit a small amount of sliding (Kooi et al., in press), making it more difficult to judge the direction of motion. Smith and Edgar (1991), using 1- and 3-cpd components, also acknowledge the occurrence of the sliding percept. Ferrera and Wilson (1990) found that the apparent direction of a partially sliding plaid could be nonveridical. (Their plaids probably exhibited some sliding due to the large differences in component orientation and temporal frequency.) This suggests the possibility that sliding may be a necessary condition for the perceived-direction error, due to a spatial frequency difference, to manifest itself. However, all observers noted that the amount of sliding did not change with eccentric viewing; if anything, the plaids became more coherent as eccentricity increased. This result argues against the suggestion that direction bias depends strongly on motion incoherence, since the size of the direction bias increased with eccentricity. ${ }^{3}$

\section{Possible Relation to Physiology}

Movshon et al. (1986) have suggested that MT is the area involved in combining the component motions (1-D) into the pattern motion (2-D) because some cells respond to pattern direction, not component direction. Furthermore, area MT receives its main input from the magno pathway (DeYoe \& van Essen, 1988), which is generally considered to be specialized for the detection and processing of motion (Livingstone \& Hubel, 1987; Zeki, 1978). If indeed MT is critical in the perception of direction, then the processes responsible for the apparent slowdown of some grating patterns and the spatial frequency interaction must occur at or before MT.

The rapid falloff of the perceived-direction and perceived-speed errors as a function of contrast shows similarities to physiological contrast saturation observed in the M pathway (Sclar, Maunsell, \& Lennie, 1990; Shapley, Kaplan, \& Soodak, 1981), as explained in detail in Stone et al. (1990). Nakayama and Silverman (1985) proposed that the psychophysical contrast saturation found in various motion tasks, such as motion aftereffects and motion hyperacuity, may be linked to this physiological saturation of cells in the magno stream. The motion errors found with both of our techniques, plaiddirection bias and speed matching, disappear near $5 \%$ contrast. If Nakayama and Silverman's hypothesis is correct, the motion system is subject to error when cells in the magno stream are not saturated. The component-to-pattern transformation thus may only operate completely correctly on input that is invariant with contrast.

Regarding the site of low-spatial-frequency dominance, Grosof (1989) examined interfrequency interactions between two components of the same orientation but with opposing motion energy in area V1. Not finding the kind needed to explain motion capture, he concluded that it must occur at a later stage in the visual pathway. ${ }^{4}$ It would therefore be interesting to examine V2 and MT cells for the presence of low- on high-spatial-frequency inhibition.

\section{REFERENCES}

Adelson, E. H., Movshon, J. A. (1982). Phenomenal coherence of moving visual patterns. Nature, 300, 523-525.

Campbell, F. W., MaffeI, L. (1981). The influence of spatial frequency and contrast on the perception of moving patterns. Vision Research, 21, 713-721.

Derrington, A. M., * Goddard, P. A. (1989). Failure of motion discrimination at high contrasts: evidence for saturation. Vision Research, 29, 1767-1776.

Derrington, A., * Suero, M. (1991). Motion of complex pattems is computed from the perceived motions of their components. Vision Research, 31, 139-149.

De Valois, K. K., De Valots, R. L., Yund, E. W. (1979). Responses of striate cortex cells to grating and checkerboard patterns. Journal.of Physiology (London), 291, 483-505.

De VAlois, K. K., ToOTElL, R. B. H. (1983). Spatial-frequencyspecific inhibition in cat striate cortex cells. Joumal of Physiology (London), 336, 359-376.

De Valois, R. L., \& DE VAlols, K. K. (1988). Spatial vision. Oxford: Oxford University Press.

DeYoe, E. A., \& VAN Essen, D. C. (1988). Concurrent processing streams in monkey visual cortex. Trends in Neuroscience, 11, 219-226.

Diener, H. C., Wist, E. R., Dichgans, J., Brandt, Th. (1976) The spatial frequency effect on perceived velocity. Vision Research, 16, 169-176.

Duncker, K. (1938). Induced motion. In W. Ellis (Ed.), Source book of Gestalt psychology (pp. 161-172). New York: Humanities Press.

Fennema, C. L., Thompson, W. B. (1979). Velocity determination in scenes containing several moving images. Computer Graphics \& Image Processing, 9, 301-315.

Ferrera, V. P., \& Wilson, H. R. (1987). Direction specific masking 
and the analysis of motion in two dimensions. Vision Research, 27, $1783-1796$.

Ferrera, V. P., * Wilson, H. R. (1990). Perceived direction of moving two-dimensional patterns. Vision Research, 30, 273-288.

Ferrera V. P., Wilson, H. R. (1991). Perceived speed of moving plaids. Vision Research, 31, 877-893.

Finney, D. J. (1971). Probit analysis. Cambridge University Press. Grosof, D. H. (1989). Studies of motion capture. Unpublished doctoral dissertation, University of California, Berkeley.

HeEger, D. J. (1987). Model for the extraction of image flow. Journal of the Optical Society of America A, 4, 1455-1470.

Keck, M. J., Palella, T. D., \& Pantle, A. (1976). Motion aftereffect as a function of the contrast of sinusoidal gratings. Vision Research, 16, 187-191.

Koor, F. L., \& DE VALois, K. K. (1992). The role of color in the motion system. Vision Research, 32, 657-668.

KoOI, F. L., De Valois, K. K., Grosof, D. H., \& De Valois, R. L. (1988). Spatial frequency affects perceived direction of a moving plaid. OSA Annual Meeting, Technical Digest Series, 11, 141.

Kool, F. L., De Valois, K. K., Switkes, E., \& Grosof, D. H. (in press). Higher-order factors influencing the perception of sliding and coherence of a plaid. Perception.

Kool, F. L., De Valois, R. L., \& Wyan, T. K. (1988). Perceived direction of moving plaids. Investigarive Ophthalmology \& Visual Science, 29(Suppl.), 265.

Krauskopf, J., Farell, B. (1990). Influence of color on the perception of coherent motion. Nature, 348, 328-331.

Livingstone, M. S., \& Hubel, D. H. (1987). Psychophysical evidence for separate channels for the perception of form, color, movement, and depth. Joumal of Neuroscience, 7, 3416-3468.

McKee, S. P., Silverman, G. H., \& Nakayama, K. (1986). Precise velocity discrimination despite random variations in temporal frequency and contrast. Vision Research, 26, 609-619.

Movshon, J. A., Adelson, E. H., Gizzi, M. S., Newsome, W. T. (1986). The analysis of moving visual patterns. Experimental Brain Research, 11(Suppl.), 117-151.

Nakayama, K., \&ilverman, G. H. (1985). Detection and discrimination of sinusoidal grating displacements. Journal of the Optical Society of America A, 2, 267-274.

Perrone, J. A. (1990). Simple technique for optical flow estimation. Journal of the Optical Society of America A, 7, 264-278.

RAmaChaNDRAN, V. S. (1987). Visual perception of surfaces: A biological theory. In S. Petry \& G. E. Meyer (Eds.), The perception of illusory contours (pp. 93-108). New York: Springer Verlag.

Ramachandran, V. S., Cavanagh, P. (1987). Motion capture anisotropy. Vision Research, 27, 97-106.

Rodman, H. R., Albright, T. D. (1989). Single-unit analysis of pattern-motion selective properties in the middle temporal visual area (MT). Experimental Brain Research, 75, 53-64.

Rodman, H. R., Gross, C. G., \& Albright, T. D. (1989). Afferent basis of visual response properties in area MT of the macaque. I. Effects of striate cortex removal. Joumal of Neuroscience, 9, 2033-2050.

Sclar, G., Maunsell, J. H. R., Lennie, P. (1990). Coding of image contrast in central visual pathways of the macaque monkey. $\mathrm{Vi}$ sion Research, 30, 1-10.

Shapley, R., Kaplan, E., SoOdak, R. (1981). Spatial summation and contrast sensitivity of $X$ and $Y$ cells in the lateral geniculate nucleus of the macaque. Nature, 292, 543-545.
Smith, A. T., Edgar, G. K. (1990). The influence of spatial frequency on perceived temporal frequency and perceived speed. Vision Research, 30, 1467-1474.

Smith, A. T., EDgar, G. K. (1991). Perceived speed and direction of complex gratings and plaids. Journal of the Optical Society of America $A, 8,1161-1171$.

Stone, L. S. \& Thompson, P. (1992). Human speed perception is contrast dependent. Vision Research, 32, 1535-1549.

Stone, L. S., Watson, A. B., \& Mulligan, J. B. (1990). Effect of contrast on the perceived direction of a moving plaid. Vision Research, 30, $1049-1068$.

Switkes, E., Bradley, A., \& De Valois, K. K. (1988). Contrast dependence and mechanisms of masking interactions among chromatic and luminance gratings. Journal of the Optical Society of America A, $5,1149-1162$.

Thompson, P. (1982). Perceived rate of movement depends on contrast. Vision Research, 22, 377-380.

WELCH, L. (1989). The perception of moving plaids reveals two motionprocessing stages. Nature, 337, 734-736.

WelCh, L., \& Bowne, S. F. (1990). Coherence determines speed discrimination. Perception, 19, 425-435.

ZEKI, S. M. (1978). Functional specialization in the visual cortex of the rhesus monkey. Nature, 274, 423-428.

\section{NOTES}

1. Threshold was measured at $3 \mathrm{~Hz}$. As the direction of movement of a plaid is changed, one component increases and the other decreases in speed. Consequently, the measured contrast sensitivities would be slightly off, the inaccuracy depending on the direction of motion of the plaid and on the slope of the contrast sensitivity function near our stimulus conditions. We chose conditions such that the range of plaid directions was small and the errors in contrast sensitivity were negligible.

2. For some experiments, a slightly different technique was used. Rather than presenting one of the two mirror versions of the plaid and asking toward which quadrant it appeared to move, both versions were presented in succession and the subject was asked toward which quadrant the second plaid moved relative to the first plaid. The order of the plaid presentations was randomized. The main advantage of this technique is that the subject is required to compare only the perceived directions of two plaids rather than the direction of the plaid relative to an external frame. We did not find significant differences in the results from these two techniques.

3. The observed changes in plaid-direction bias at $10^{\circ}$ eccentricity, but not at $5^{\circ}$, could be due to changes in contrast sensitivity with eccentricity (Figure 7 ). At $10^{\circ}$, the contrast sensitivity of both frequencies drops and the relative sensitivity changes in favor of the lower spatial frequency component. The latter effect should increase the low-spatial-frequency dominance. At $5^{\circ}$, the relative contrast sensitivity remains the same, yet a significant increase in low spatial frequency is observed.

4. Interfrequency inhibition has been reported by, among others, K. K De Valois and Tootell (1983). However, the inhibition they reported did not show the spatial frequency asymmetry necessary to explain motion capture.

(Manuscript received September 19, 1991; revision accepted for publication April 2, 1992.) 